EUROPEAN ORGANIZATION FOR NUCLEAR RESEARCH

CERN-PPE / 97-65-Rev

August 5, 1997

\title{
Open charm contribution to dilepton spectra produced in nuclear collisions at SPS energies
}

\author{
P. Braun-Munzinger, D. Miśkowiec \\ GSI, Darmstadt, Germany \\ A. Drees \\ Universität Heidelberg, Germany \\ C. Lourenço \\ CERN, Geneva, Switzerland
}

\begin{abstract}
Measurements of open charm hadro-production from CERN and Fermilab experiments are reviewed, with particular emphasis on the absolute cross sections and on their $\mathrm{A}$ and $\sqrt{s}$ dependences. Differential $p_{\mathrm{T}}$ and $x_{\mathrm{F}}$ cross sections calculated with the PYTHIA event generator are found to be in reasonable agreement with recent data. The calculations are scaled to nucleusnucleus collisions and the expected lepton pair yield is deduced. The charm contribution to the low mass dilepton continuum observed by the CERES experiment is found to be negligible. In particular, it is shown that the observed low mass dilepton excess in S-Au collisions cannot be explained by charm enhancement.
\end{abstract}

Accepted for publication in Z. Phys. C 


\section{Introduction}

In order to understand whether the data collected in nucleus-nucleus collisions signal new physics such as quark-gluon plasma formation, there are essentially two possible procedures. The first one consists in using Monte Carlo event generators to calculate what should happen in conventional scenarios. A significant difference between prediction and experiment would indicate new physics. The standard event generator codes, however, simulate nucleus-nucleus collisions only in what concerns soft processes. Physics results based on conventional hard processes in nucleus-nucleus collisions, on the other hand, can be predicted by extrapolation from pp and pA data. This second method has been used in the study of high mass dimuon production. The present understanding of $\mathrm{J} / \psi, \psi^{\prime}$ and Drell-Yan production in pA interactions, from data collected at CERN and at Fermilab, allows to estimate the behavior expected in $\mathrm{S}$ and $\mathrm{Pb}$ induced collisions [1].

Open charm production is a hard process and the presently available nucleus-nucleus event generators cannot be used to estimate production yields or contributions to the dilepton spectra measured at SPS energies. The SPS heavy ion collaborations NA38 and HELIOS-3 have used the Pythia Monte Carlo event generator [2] to calculate charm production on the pp level and used a scaling which is linear with the nuclear mass number A to compare the estimated values to the data collected with sulphur beams [3, 4]. An initial attempt to assess the charm decay contribution to dilepton spectra at higher masses has been presented by A. Shor [5]. In this paper we use PYTHIA to evaluate whether the low mass electron pair excess observed by CERES [6] might be explained by charm enhancement in heavy ion collisions.

The theoretical understanding of the features seen in the charm data for $\mathrm{pp}$ and $\mathrm{pA}$ collisions has made substantial progress in recent years but is still facing some difficulties (see [7 for a recent review). The availability of NLO calculations has not eliminated the need for uncomfortably large values for the intrinsic transverse momentum $k_{\mathrm{T}}$ of the incoming partons (the ' $k_{\mathrm{T}}$ kick'). Adding together the uncertainty in c-quark mass $\left(m_{c}\right.$ ranging from 1.2 to $1.8 \mathrm{GeV} / c^{2}$ ), values of $\left\langle k_{\mathrm{T}}^{2}\right\rangle$ that can go up to $2 \mathrm{GeV}^{2} / c^{2}$, doubts on which fragmentation scheme better describes the hadronization process, and the ignorance of higher order effects, especially at low transverse momentum, we get the feeling that the theoretical understanding is still somewhat unclear. For a detailed discussion of these theoretical uncertainties including a complete list of references see [7]. It is not our intention

to clarify this situation. Rather we wish to evaluate whether a PYтHIA 
based model is able to describe reasonably well the available data on open charm production. If so, we can use it as a good basis to predict, in the absence of new physics, charm production in nucleus-nucleus collisions and to estimate the charm contribution to dilepton spectra.

In Section 2 we compare the results provided by PYTHIA with the recent FNAL and CERN data on open charm hadro-production, with particular emphasis on the energy dependence of the charm cross section, on the distributions in transverse momentum $p_{\mathrm{T}}$ and the Feynman $\mathrm{x}$ variable $x_{\mathrm{F}}$ and on the correlations between charmed hadrons. We then compute the lepton pair yield from simultaneous semileptonic decays of $D$ and $\bar{D}$ mesons and $\Lambda_{c}$ baryons and compare it to the experimentally detected inclusive dilepton yield in p-p and p-A collisions. Scaling the results to nucleus-nucleus collisions and comparing them to data as well as the possibility of enhanced charm production in these collisions are discussed in Section 4.

\section{Charm production in pp and pA collisions}

The recent Fermilab and CERN experiments on the production of $D$ mesons in proton and pion induced collisions provide a rather detailed picture of charm hadro-production. Fig. 11 collects the forward $\left(x_{\mathrm{F}}>0\right) D$ meson production cross sections as measured by several experiments, as a function of $\sqrt{s}$, the center of mass energy. Where appropriate, the values have been adjusted to reflect the current knowledge of branching ratios [8]. The cross section values for proton induced reactions, published by experiments NA27, E743 and E653 for all $x_{\mathrm{F}}$, were divided by 2 . The results obtained with nuclear targets were divided by the target atomic mass number A. The error bars reflect statistical and systematical uncertainties added in quadrature and include the uncertainty on the branching ratios. The order of magnitude discrepancies found in earlier measurements have disappeared and the energy dependence of the data is rather consistent with PYTHIA based QCD predictions (solid lines in Fig. 1). The calculations do not reproduce very well the observed $D^{+} / D^{0}$ ratio and thus two different up-scale factors $K$ would be needed to reproduce charged and neutral $D$ cross sections simultaneously. (See also a comment concerning this situation in [7]). Since the spectral distributions for $D^{+}$and $D^{0}\left(D^{-}\right.$and $\left.\bar{D}^{0}\right)$ are similar (see below), we use in the following an average $K$ factor of 5.0 determined to reproduce the overall $D$ yield.

The hadro-production of open charm mesons on nuclear targets has recently been precisely measured in collisions induced by $800 \mathrm{GeV}$ protons. 

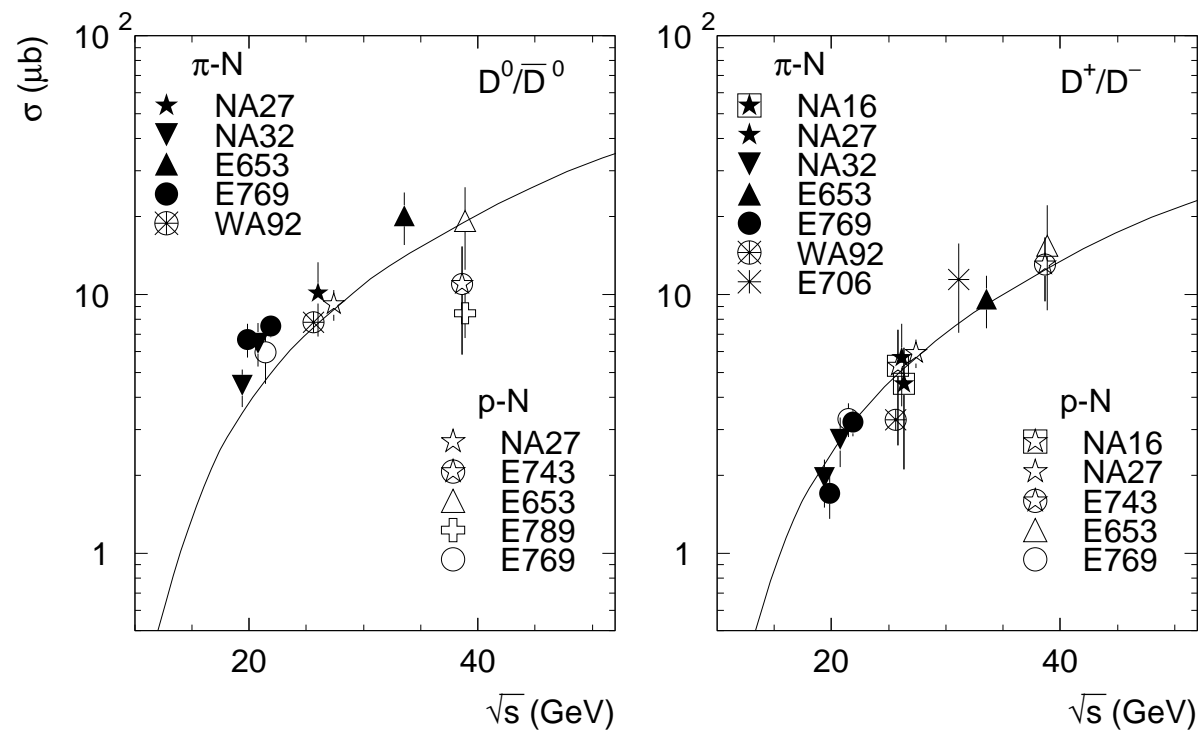

Figure 1: Cross section for forward $\left(x_{\mathrm{F}}>0\right) D^{0}, \bar{D}^{0}$ (left) and $D^{+}, D^{-}$(right) production in proton and pion induced reactions, as a function of the center of mass energy. The data were taken from NA16 [9], NA27 [10], E743 [11], E653 [12], E789 [13], E769 [14], NA32 [15], WA92 [16], and E706 [17]. The solid lines represent the pp PYTHIA calculations, up-scaled by a factor of 3.5 (left panel) and 7 (right panel).

Parameterizing the nuclear target dependence in the usual way, $\sigma(p A \rightarrow$ $D+X)=\sigma_{N} A^{\alpha}$, with $\sigma_{N}$ the open charm cross section for proton-nucleon collisions, the E789 experiment has obtained $\alpha=1.02 \pm 0.03 \pm 0.02$, for $0.0<x_{\mathrm{F}}<0.08$ (average $x_{\mathrm{F}}$ of 0.031), comparing Be and Au targets 13. With $250 \mathrm{GeV} \pi^{+}$and $\pi^{-}$beams, the E769 experiment has measured $\alpha=1.00 \pm 0.05 \pm 0.02$ for $D$ mesons with $x_{\mathrm{F}}$ between 0.0 and 0.8 , using a target system composed of $\mathrm{Be}, \mathrm{Al}, \mathrm{Cu}$ and $\mathrm{W}$ foils 18 . Besides, E769 has not found any significant dependence of $\alpha$ on $p_{\mathrm{T}}$ or $x_{\mathrm{F}}$, at least for the $x_{\mathrm{F}}<0.5$ region, responsible for most of the cross section. These findings confirm what one expects for a hard process, in which the partons in the nuclear target independently contribute to the total cross section.

In the following we will use hadron-hadron and hadron-nucleus data, along with the atomic mass number dependence, to evaluate to which extent charm production is described by leading order QCD calculations. To this end we have performed calculations with the code PYTHIA using MRS G 
parton distributions $\left[19\right.$ and $\left\langle k_{\mathrm{T}}^{2}\right\rangle=1 \mathrm{GeV}^{2} / c^{2}$. We checked that using $\left\langle k_{\mathrm{T}}^{2}\right\rangle=2 \mathrm{GeV}^{2} / c^{2}$ changes the dilepton yield by less than $10 \%$. Most of the calculations were performed for proton-proton collisions. We have, however, checked that isospin dependences are small for open charm production. At $\sqrt{s}=18 \mathrm{GeV}$ the differences predicted by PYTHIA in D-meson distributions from proton-proton and neutron-neutron collisions are below $10 \%$. Since open charm production is mostly due to gluon fusion this is not unexpected.
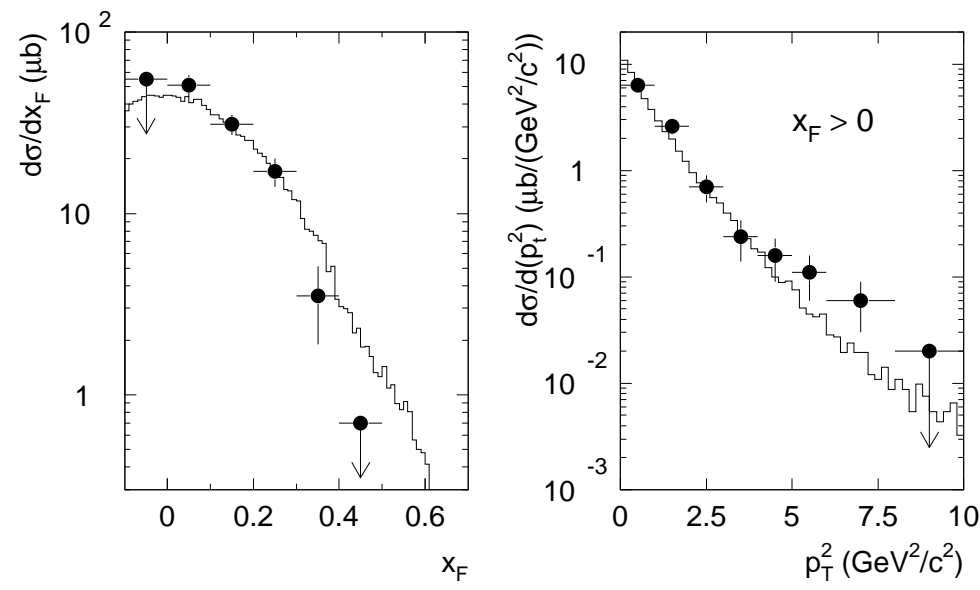

Figure 2: $D^{+}, D^{-}, D^{0}, \bar{D}^{0}, D_{s}^{+}, D_{s}^{-}$production in p-p at $250 \mathrm{GeV}$ : comparison between experiment 20] (black dots) and the up-scaled PYTHIA calculation (histograms).

The energy dependence of charm production is rather well reproduced by these calculations (Fig. 11). In Fig. 2 we present a comparison of PYTHIA predictions for $p_{\mathrm{T}}$ and $x_{\mathrm{F}}$ distributions with data from the E769 collaboration at a beam energy of $250 \mathrm{GeV}$ [20]. The absolute normalization of the calculations has been adjusted to reproduce the $D$ meson data $\left(D^{+}\right.$, $D^{-}, D^{0}, \bar{D}^{0}, D_{s}^{+}, D_{s}^{-}$), yielding an average $K$ factor of 5.0, which is used for all subsequent calculations, independent of beam momentum. Notice that the value of $K$ depends on the $c$-quark mass used; here we have used $m_{c}=1.35 \mathrm{GeV} / c^{2}$. It also depends on the choice of the $Q^{2}$ scale used in PythiA. In our calculations we have used $Q^{2}=\hat{s}$, the parton-parton center of mass energy squared. We have verified that, if the $Q^{2}$ scale is given by $\hat{m}_{T}^{2}$ (PYTHIA's default), the calculated cross sections increase by a factor of two, implying a corresponding decrease in the $K$ factor. Here $\hat{m}_{T}$ is the transverse mass of the outgoing particles produced in the hard scattering. 
Note that the kinematical distributions do not depend appreciably on the choice of the $Q^{2}$ scale.

Apart from the absolute normalization, the PYTHIA calculations reproduce reasonably well the measured $p_{\mathrm{T}}$ and $x_{\mathrm{F}}$ dependence, implying that the basic kinematics of the charm production process is correctly simulated.

The agreement with the data points can be further improved by using a $\delta$-function for the fragmentation of the charmed quarks in the charmed mesons. However, we find it difficult to believe that a $\delta$-function can be a good description of the hadronization process and, therefore, have used,
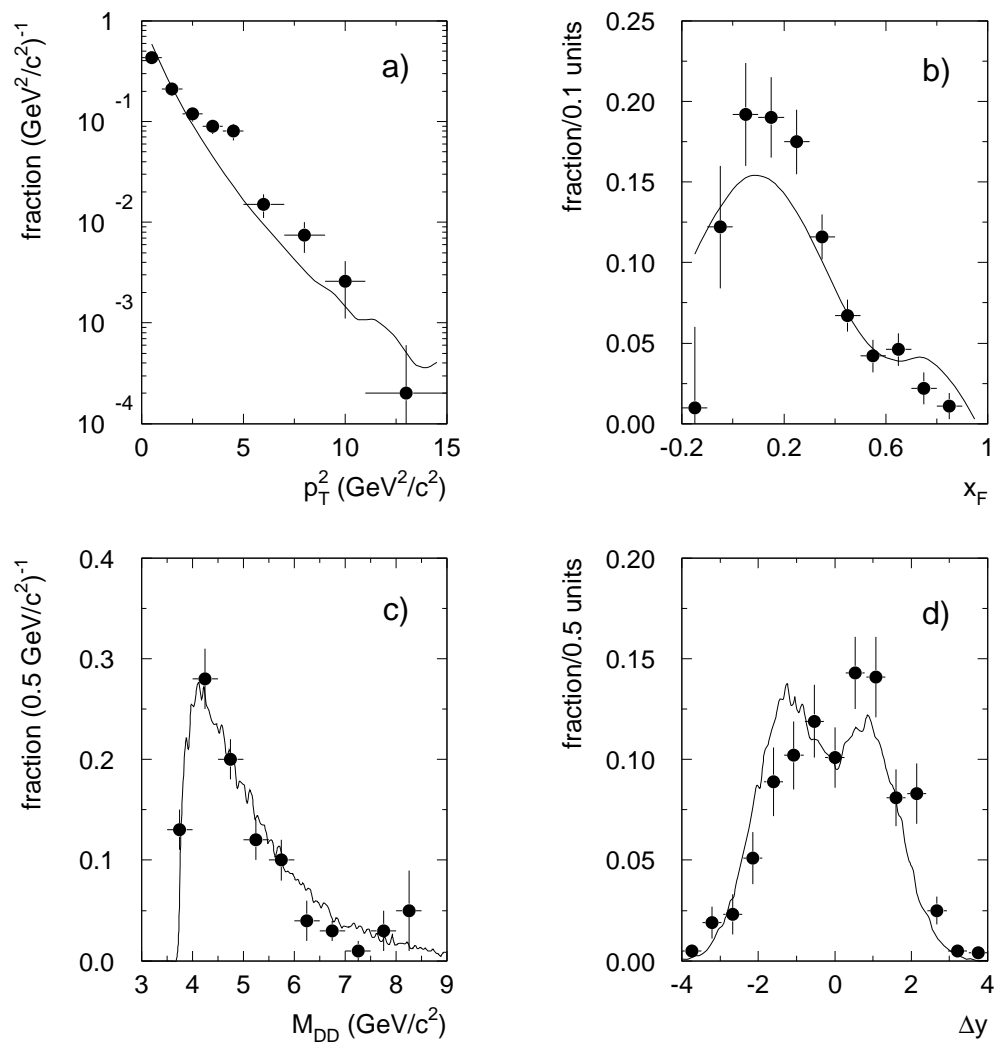

Figure 3: $\quad D \bar{D}$ data from $\pi^{-}-\mathrm{Cu}$ collisions at $\sqrt{s}=26 \mathrm{GeV}$ 16. Parts a) and b) represent the $p_{\mathrm{T}}^{2}$ and $x_{\mathrm{F}}$ distributions of the $D \bar{D}$ pair. Parts c) and d) show the $D \bar{D}$ invariant mass and the rapidity difference $\Delta y=y_{D}-y_{\bar{D}}$ distributions. The data are compared to the PyтHIA calculation (solid line). The PүтніA distributions are normalized in the same way as the data, i.e. such that the integral over the displayed range is unity. 
in the calculations reported in this paper, the Lund string fragmentation model (PYTHIA's default).

To further check the kinematics of the charm production process as implemented in PyтнIA we present in Fig. 3 comparisons with the WA92 data for $\pi^{-}-\mathrm{Cu}$ interactions, where kinematical correlations between charmed particles were measured at $\sqrt{s}=26 \mathrm{GeV}$ [16]. Fig. 3a shows good agreement between the transverse momentum spectrum calculated with PYTHIA and the data. As shown in Figs. 3b, 3c, and 3d, also the Feynman x distribution of the $D \bar{D}$ pair, the distribution in $D \bar{D}$ invariant mass $M_{D \bar{D}}$, as well as the rapidity difference between $D^{+}\left(D^{0}\right)$ and $D^{-}\left(\bar{D}^{0}\right)$ are reasonably well reproduced by the PYTHIA calculations, lending considerable confidence to the accuracy of the calculated lepton pair mass spectra.
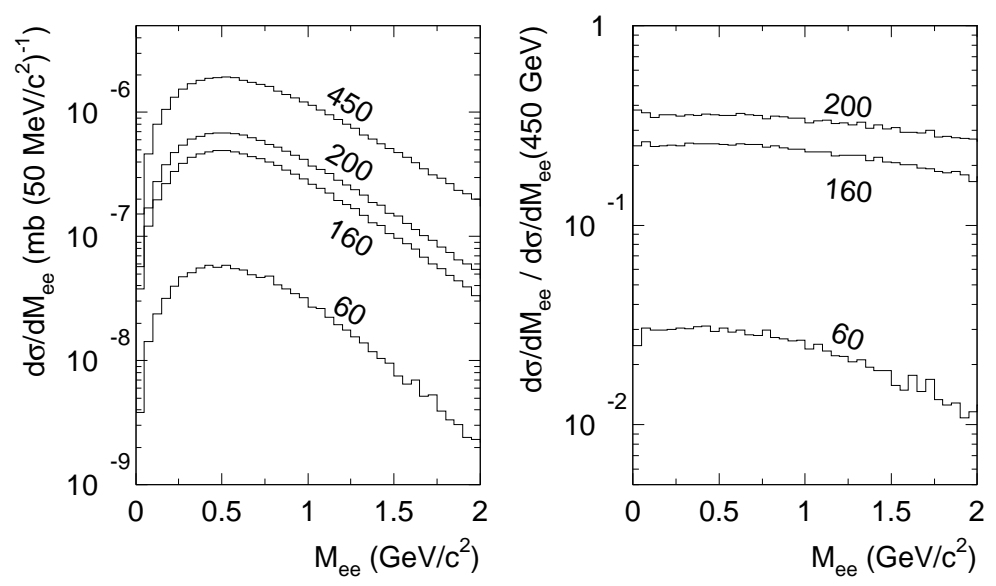

Figure 4: Left: Invariant mass spectra of dielectrons from charm decay in pp collisions for different beam momenta (PYTHIA). Right: The same normalized to the spectrum at $450 \mathrm{GeV}$.

To complete the systematics, and to lead into the discussion of charm contributions to dilepton spectra, we present in Fig. 4 the di-electron mass distributions from semi-leptonic $D$-decays, without any phase space selection, for various beam energies. The calculated mass distributions are all rather broad, with a shallow maximum near $M_{e^{+}} e^{-}=0.5 \mathrm{GeV} / c^{2}$. No strong energy dependence is observed. To emphasize this point we show, in the right hand part of Fig. 4, the ratio of mass distributions relative to the one computed at $450 \mathrm{GeV}$. From this plot one observes only a slight narrowing of the mass distributions with decreasing beam momentum. Es- 
pecially in the mass range $0<M_{e^{+}} e^{-}<1 \mathrm{GeV} / c^{2}$, the variation of the ratio with mass is less than $20 \%$.

The shape of the lepton pair mass distributions depends crucially on the implementation in PyтHIA of semi-leptonic $D$ decays. In order to check if such decays are treated correctly we compare, in Fig. 5, the momentum distribution of electrons from $D$ decays in PYTHIA with experimental distributions [21]. The theoretical distributions, where modes with $\mathrm{K}$ mesons and with $\mathrm{K}^{*}$ mesons contribute with roughly equal weight, are in good agreement with the measured spectrum. Furthermore, we have verified that the values used in PyтнiA for the semileptonic decay branching ratios are in excellent agreement with the most recent values of [8]. We conclude, therefore, that uncertainties in the lepton energy spectra and branching ratios from $D$ meson decays can be neglected in the following discussion.

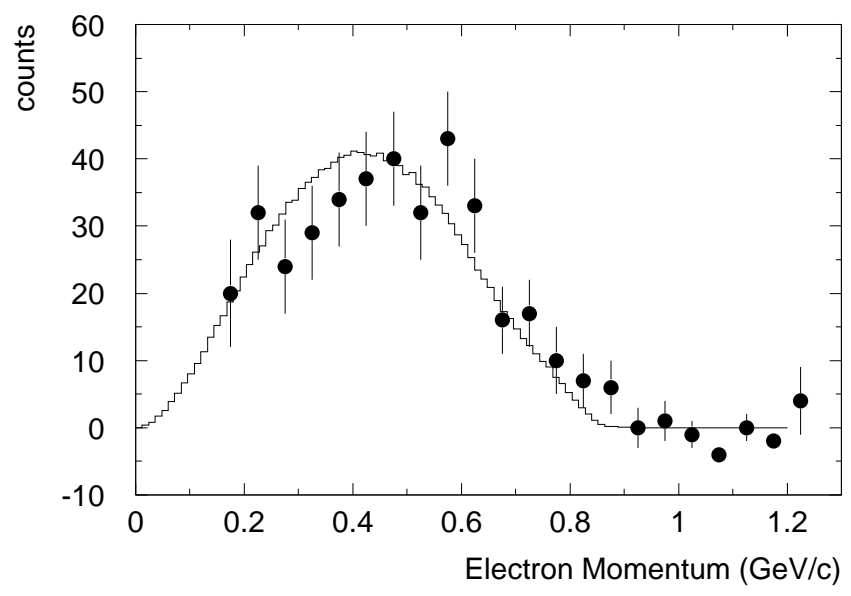

Figure 5: Momentum distribution of electrons from $D^{+}$and $D^{0}$ decays, measured in the rest frame of the decaying mesons. The experimental data (full dots) were taken from ref. [21]. The solid histogram was extracted from the Pythia calculation.

The shape of the dielectron mass spectrum also depends on the shape of the $D \bar{D}$ invariant mass spectrum. In 1983, Fischer and Geist 22] proposed a scaling curve for the ratio between the width of the parent mass distribution and the threshold mass $m^{\text {thr }}=2 m_{\mathrm{D}}$, plotted against the ratio between threshold mass and $\sqrt{s}$. Here, $m_{\mathrm{D}}$ is the mass of the $D$ meson. This curve was motivated by ISR data and by early QCD calculations 23. In Fig. 6 we compare it with the present PүтніA calculations. Although a trend 


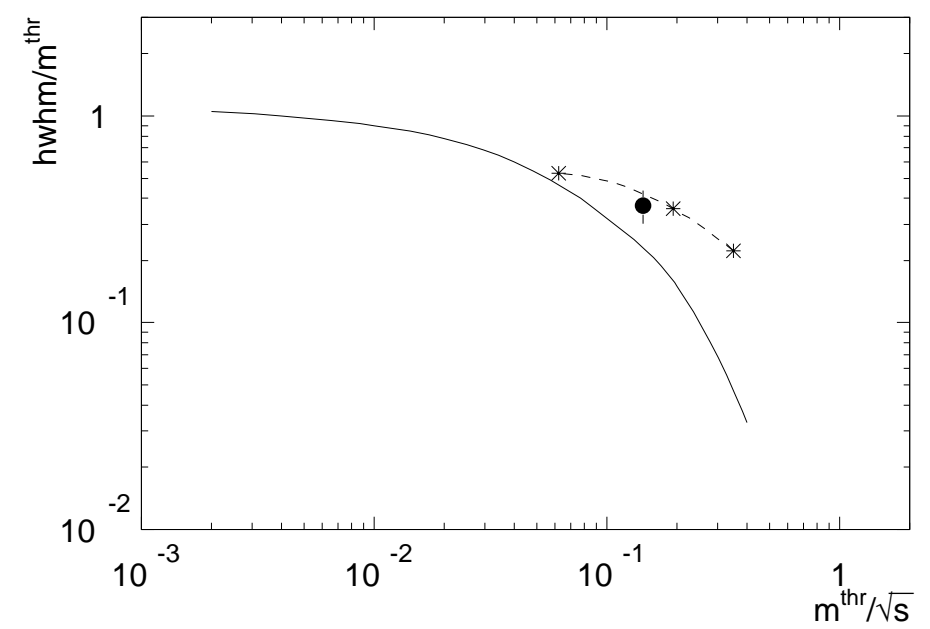

Figure 6: Relation between the collision energy and the half width at half maximum of the $M_{D \bar{D}}$ distribution, as proposed by Fischer and Geist (solid line) and as calculated by PYтHIA for pp (stars connected by dashed line). Black dot: WA92 measurement [16].

similar to the Fischer-Geist curve emerges from the present calculations, the detailed shape looks quite different. We note, however, that the WA92 measurement (black dot in Fig. 6) is in much better agreement with the present calculations than with the Fischer-Geist scaling.

\section{Dilepton yield from associated charm produc- tion in proton induced collisions}

In the previous section we have shown that calculations with the code PYтнiA describe reasonably well the most recent data on $x_{F}$ and $p_{\mathrm{T}}$ distributions of $D$ mesons as well as rapidity correlations and invariant mass spectra of the $D \bar{D}$ pairs [16,20] in the center-of-mass energy regime of $\sqrt{s} \sim 20 \mathrm{GeV}$. In addition, the characteristics of the semi-leptonic decays of $D$ mesons are correctly reproduced. This gives us confidence that the level of lepton pair yield from charm production can be reliably calculated for pp and $\mathrm{pA}$ collisions on the basis of a PYTHIA simulation. We will now compare our estimate of the lepton pair yield from charm production to data on the inclusive lepton pair yield. Both leptons from $D$ meson and from $\Lambda_{c}$ decays 
are included in the calculations.

First we discuss the results of Chilingarov et al. [24] who have measured $e^{+} e^{-}$pairs in pp collisions at the $\operatorname{ISR}(\sqrt{s} \approx 60 \mathrm{GeV})$. For this energy PYTHIA (including the above discussed $K$ factor) predicts a $c \bar{c}$ cross section of $100 \mu \mathrm{b}$, roughly in agreement with the value discussed in [24]. The $e^{+} e^{-}$ data of Chilingarov et al. and our calculation for $e^{+} e^{-}$pairs from open charm are shown in Fig. 7 . We have applied the cut $p_{\mathrm{T}}>1.6+0.5 M_{e^{+}} e^{-}$ (with $p_{\mathrm{T}}$ and $M_{e^{+}} e^{-}$in $\mathrm{GeV}$ ) on the electron pair transverse momentum in order to simulate the experimental cuts (see Fig. 5 in [24]).

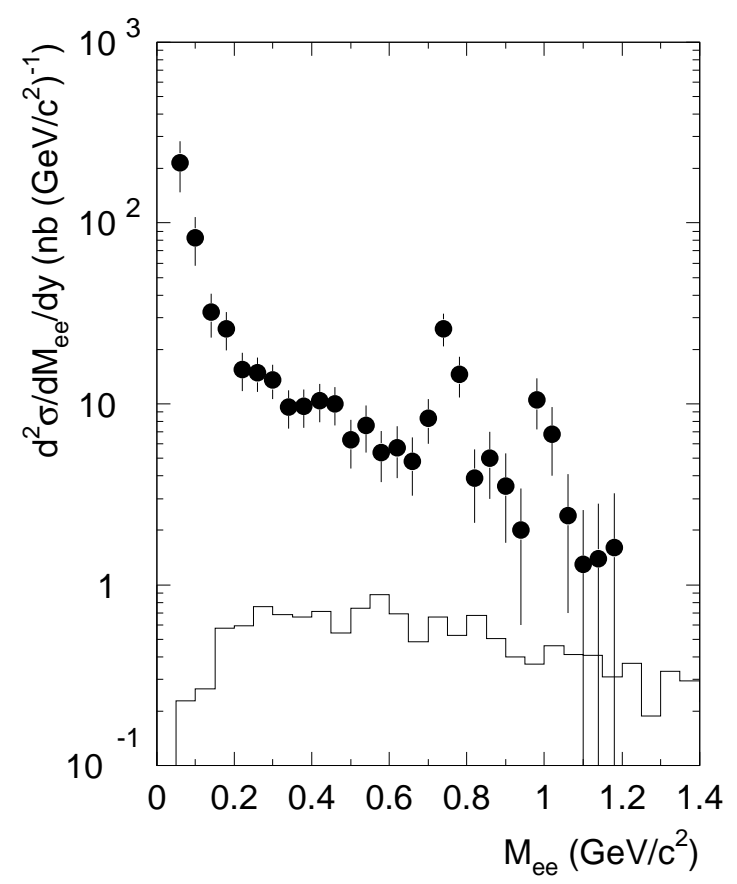

Figure 7: Invariant mass distribution for $e^{+} e^{-}$pairs from pp collisions at $\sqrt{s}=60 \mathrm{GeV}$, measured at mid-rapidity. The solid line represents the $e^{+} e^{-}$ pairs from charm decays, as predicted by our PYTHIA calculation.

According to the present calculations, and contrary to the conclusions of [24], the contribution from charm decays is approximately one order of magnitude below the observed dilepton yield while the shape of our computed $e^{+} e^{-}$mass spectrum is rather similar to that displayed in Fig. 6 of ref. 24]. In order to check whether this result depends on the value of $\left\langle k_{\mathrm{T}}^{2}\right\rangle$ used in the simulation, we repeated the procedure with $\left\langle k_{\mathrm{T}}^{2}\right\rangle=2 \mathrm{GeV}^{2} / c^{2}$. 
The corresponding yield was higher by only about $30 \%$. Of similar size are the uncertainties due to the (small) differences between calculated and measured $x_{\mathrm{F}}$ distributions for $D$ mesons (see Fig. 2). We assume that the difference between our results and the conclusions of Chilingarov et al is connected with the fact that, in ref. [24], the yield for $e^{+} e^{-}$pairs was calculated assuming isotropic decay in the pair rest frame, while PYTHIA incorporates a strong anisotropy. In addition, in ref. [24], it was assumed that the lepton pairs are produced with uniform rapidity distribution. The fact that PYTHIA reproduces well the observed distribution of rapidity gaps between the $D \bar{D}$ mesons (see Fig. 3), which is at the root of the anisotropy, lends strong support to the validity of the present calculations. We therefore conclude that, at ISR energies, the charm decay contribution to the dilepton continuum below the $\phi$-mass is small. To explain the observed continuum one should probably revisit the Dalitz decay contributions to the mass spectra.
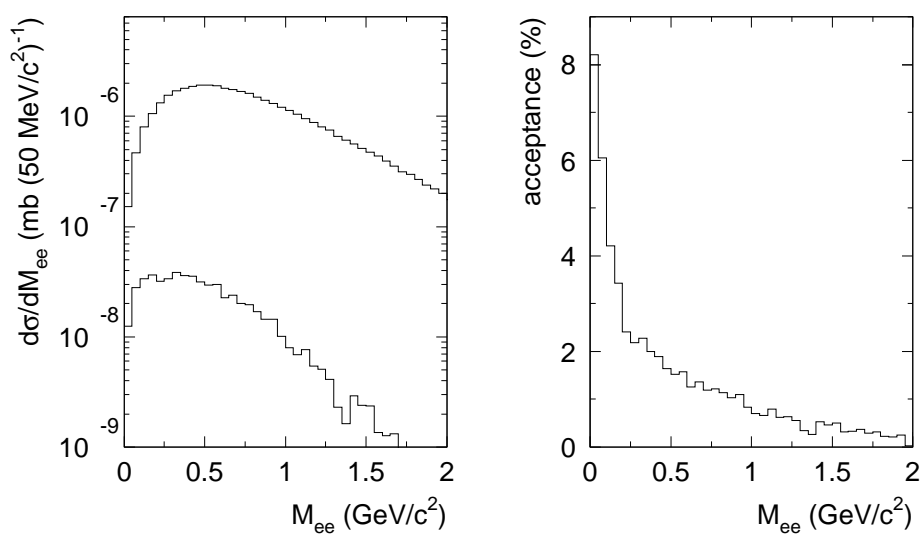

Figure 8: Left: Invariant mass spectra of dielectrons from charm decays in p-p collisions at beam energy $450 \mathrm{GeV}$ (РҮтнIA), before (upper histogram) and after (lower histogram) applying the CERES filter. Right: the ratio of the two histograms.

Recently the CERES/NA45 experiment has measured $e^{+} e^{-}$pairs in p-Be and p-Au collisions at $450 \mathrm{GeV} / c$ incident proton momentum [6]. In order to compare our Pythia calculation to the CERES data, we included in our simulation their momentum resolution and kinematic cuts $\left(p_{\mathrm{T}}>50 \mathrm{MeV} / c\right.$ and $2.1<\eta<2.65$ for electrons and positrons). The effect of the CERES acceptance on the $e^{+} e^{-}$mass spectra from charm decays is severe, as can be seen from Fig. 8, where we have compared $e^{+} e^{-}$mass distributions from 
charm before and after applying the acceptance cut. The integral of the mass distribution is reduced by approximately a factor of 60 and, in addition, the shape is distorted, leading to a steeper mass dependence for large pair masses. The dependence of the cross section on the nuclear mass number was taken into account as outlined in the next section. Finally the cross section was converted into a multiplicity distribution and scaled by $<\mathrm{d} N_{c h} / \mathrm{d} y>^{-1}$ taken from the CERES experiment which gives the same normalization as used by the CERES collaboration.

Fig. 9 shows the CERES data as published in ref. [6], but including our estimate of the contribution from charm production. Clearly, also in pnucleus collisions charm production does not give an important contribution to the dilepton continuum below the light vector mesons.

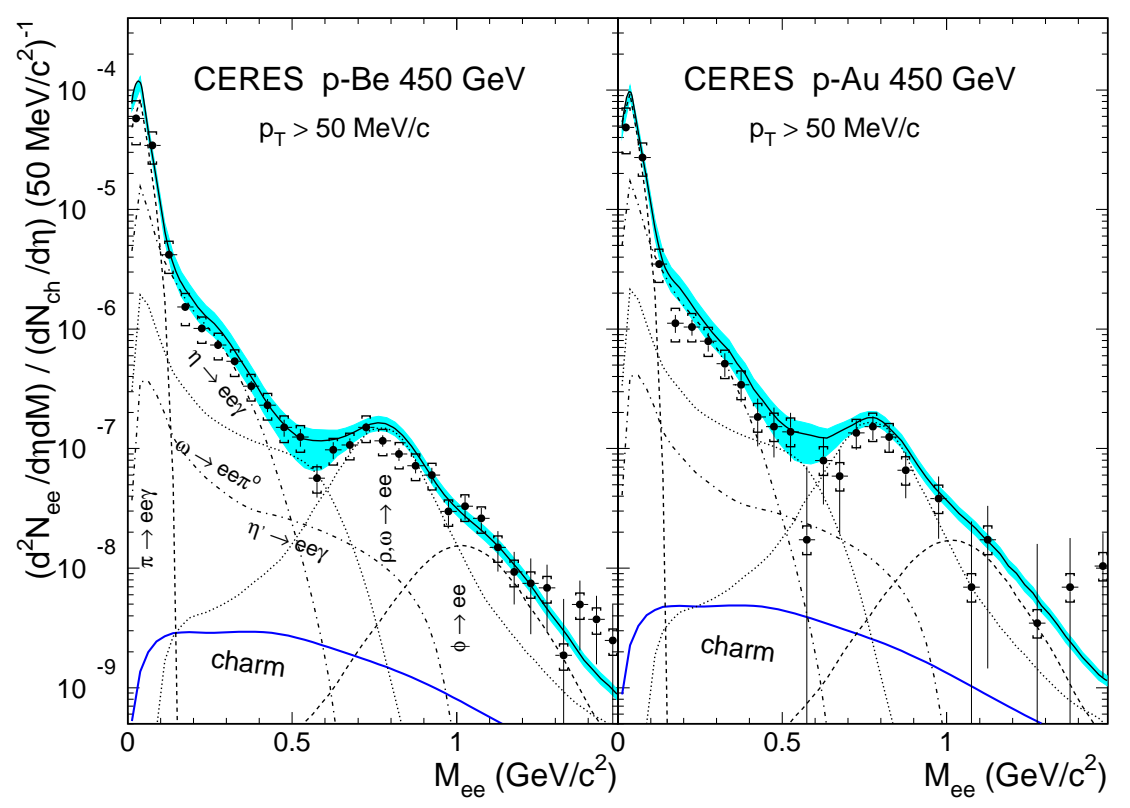

Figure 9: Invariant $e^{+} e^{-}$mass distribution from $450 \mathrm{GeV} / c$ p-Be and p-Au collisions measured by the CERES experiment. The background from associated charm production has been added to the hadron decay contribution according to our estimate.

HELIOS/NA34-1 25] has published a measurement of $e^{+} e^{-}$and $\mu^{+} \mu^{-}$ pairs in p-Be collisions at $450 \mathrm{GeV} / c$ momentum for the same mass region. From their analysis one concludes that the charm decay contribution to their data is negligible. 
While lepton pairs from associated charm production can be neglected for low invariant masses, for masses above the $\phi$-meson the situation is quite different. Two experiments - NA38 and HELIOS/NA34-3 - have measured muon pairs from p-nucleus collisions in this mass range. Both experiments have shown that the dimuon cross section in $\mathrm{p}-\mathrm{W}$ collisions at $200 \mathrm{GeV} / c$ can be accounted for by charm production and pairs produced via the Drell-Yan mechanism. The HELIOS-3 estimate [26] of the charm cross section was based on an approach similar to the one presented in this paper. The experiment NA38 used PYTHIA to determine the shape of the dilepton background from charm production but fitted the charm cross section to their muon pair data [3]. The result is compatible with the cross sections shown in Fig. 1. Both experiments consistently show that, for masses above the $\phi$ meson, $c \bar{c}$-production is an important source of lepton pairs until the continuum is overwhelmed by pair production via the DrellYan mechanism in the mass region beyond the $\mathrm{J} / \psi$.

\section{Extrapolating to nucleus-nucleus collisions}

Since the charm cross section scales with the number of target nucleons going from p-p to p-nucleus collisions, we scale the p-p calculations from PYтнIA with the total number of nucleon-nucleon collisions to obtain the nucleusnucleus cross section. As discussed above, isospin effects can be neglected for the calculation of open charm production within an accuracy of better than $10 \%$. The total number of nucleon-nucleon collisions is calculated from the nuclear collision geometry based on the concept of an overlap function $T_{A B}(b)$ for $A+B$ collisions at impact parameter $b$ [27]. This leads to:

$$
\frac{\mathrm{d} \sigma}{\mathrm{d} y}^{A B}=\frac{\mathrm{d} \sigma^{p p}}{\mathrm{~d} y} \int_{b_{1}}^{b_{2}} \mathrm{~d}^{2} b T_{A B}(b) .
$$

Here, the integration limits indicate the impact parameter range covered in the experiment. For minimum bias collisions, where $b_{1}=0$ and $b_{2}=\infty$ this leads to

$$
{\frac{\mathrm{d} \sigma^{A B}}{\mathrm{~d} y}}^{A B}=A B{\frac{\mathrm{d} \sigma^{p p}}{\mathrm{~d} y}}^{p p}
$$

For the rapidity density distributions this implies

$$
\frac{\mathrm{d} N}{\mathrm{~d} y}^{A B}=\frac{\mathrm{d} N}{\mathrm{~d} y}^{p p} \sigma_{\text {inel }}^{p p} \frac{\int_{b_{1}}^{b_{2}} d^{2} b T_{A B}(b)}{\pi\left(b_{2}^{2}-b_{1}^{2}\right)} .
$$


Here, $\sigma_{\text {inel }}^{p p}$ is the inelastic nucleon-nucleon cross section at the appropriate energy巴. Equation 3, when applied to central collisions $(b \approx 0)$ and using a sharp sphere approximation of the nuclear density profile for the evaluation of the overlap function, results in the familiar dependence

$$
\frac{\mathrm{d} N}{\mathrm{~d} y}^{A A}(b \sim 0) \approx \frac{\mathrm{d} N}{\mathrm{~d} y}^{p p} A^{4 / 3} .
$$

We note that this approach neglects possible effects due to the Fermi motion of the nucleons in the colliding nuclei. However, the threshold for open charm production is $\sqrt{s}=5.5 \mathrm{GeV}$ compared to the $\sqrt{s}=17.3 \mathrm{GeV}$ available in $\mathrm{Pb}-\mathrm{Au}$ collisions so that Fermi motion effects are not expected to play an important role. We use Woods-Saxon distributions for the nuclear densities [28] to evaluate the thickness function $\mathrm{T}_{\mathrm{AB}}(\mathrm{b})$ ( or $\mathrm{T}_{\mathrm{A}}$ for the case of $\mathrm{pA}$ collisions) and adjust the impact parameter range to obtain the fraction of inelastic cross section sampled in each experiment. Applying this procedure to the pp results from PyThia we obtain the $D$ meson yield expected for nucleus-nucleus collisions. In Fig. 10 we show the prediction for the rapidity distribution of $D$ mesons in central ( $5 \%$ of geometrical cross section) $\mathrm{Pb}+\mathrm{Pb}$ collisions at $160 \mathrm{GeV} / c /$ nucleon. The distributions exhibit rather drastic differences between $D$ mesons and their anti-particles.

These differences originate, within the PYTHIA framework, from the interaction of $c$ and $\bar{c}$ quarks with valence quarks of the colliding nucleons which takes place prior to hadronization. While the $\bar{c}$ forms a string with a single valence quark, the $c$ interacts with valence diquarks, and is dragged towards beam or target rapidity. As a consequence, the $D^{+}$and $D^{0}$ meson rapidity distributions are broadened and, at energies around $\sqrt{s}=20 \mathrm{GeV}$, even exhibit minima at midrapidity.

Similar asymmetries, although different in detail because of the different quark content of the projectile, have recently been observed for $\pi^{-}$nucleus interactions at $500 \mathrm{GeV} / c$ [29]. There it is also shown how well these asymmetries are reproduced by PYTHIA It would be interesting to verify experimentally whether such asymmetries also exist in proton induced collisions, in particular at $\sqrt{s} \sim 20 \mathrm{GeV}$, since according to PүтніA these asymmetries tend to disappear at higher beam energies.

\footnotetext{
${ }^{1}$ In actual calculations the inelastic nucleon-nucleon cross section drops out as we compute directly $\frac{\mathrm{d} \sigma}{\mathrm{d} y}^{p p}=\frac{\mathrm{d} N}{\mathrm{~d} y}^{p p} \sigma_{\text {inel }}^{p p}$.

${ }^{2}$ In ref. [29] it is shown that the measured asymmetries can be well reproduced by PyтhiA if one sets the mass of the charmed quark to $1.7 \mathrm{GeV} / c^{2}$ and otherwise uses the same parameters as are used in the present investigation. With our parameter set the agreement between data and calculations is only slightly deteriorated.
} 


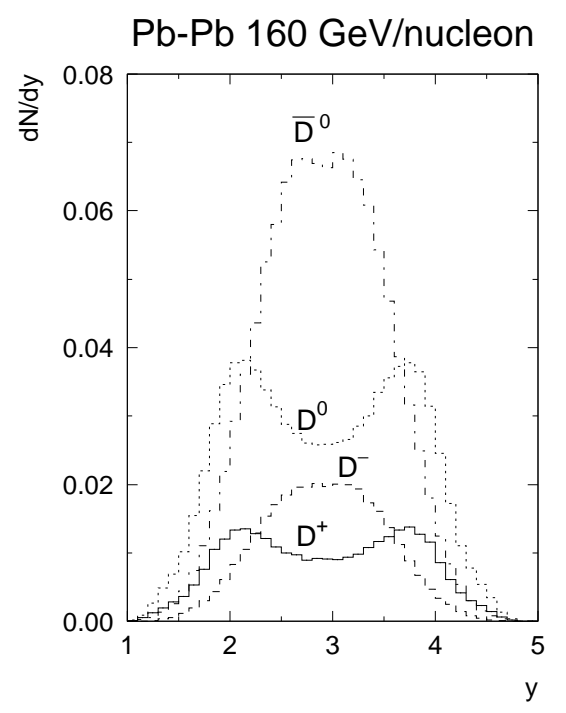

Figure 10: Calculated rapidity distributions of $D^{+}, D^{-}, D^{0}, \bar{D}^{0}$ from central $(5 \%) \mathrm{Pb}+\mathrm{Pb}$ collisions at $160 \mathrm{GeV} / c /$ nucleon. The ordinate represents absolute multiplicities per unit rapidity.

The shapes of the $D$ meson rapidity distributions for $\mathrm{Pb}+\mathrm{Pb}$ collisions might also be distorted in the nuclear environment, as observed for $p_{\mathrm{T}}$ distributions measured in p-nucleus collisions, commonly referred to as Cronin effect 30]. Clearly, a direct measurement of $D$ meson production in $\mathrm{Pb}+\mathrm{Pb}$ collisions would be of great interest. The possible modifications discussed above should not change the total rate significantly and we expect that (in the absence of charm enhancement) the rapidity density of $D$ mesons from $\mathrm{Pb}+\mathrm{Pb}$ collisions at $160 \mathrm{GeV} /$ nucleon is $\mathrm{d} N / \mathrm{d} y=0.10-0.15$ at midrapidity.

As in the previous section we want to focus on the charm decay contribution to the inclusive lepton pair production. In Fig. 11 the results of our calculation are compared to the S-Au $e^{+} e^{-}$data published by the CERES group [6]. Clearly, charm production in the "standard" scenario as presented here contributes less than one percent of the observed dilepton yield. The slight difference in shape of the charm contribution compared to Fig. 9 is solely due to the higher $p_{\mathrm{T}}$-cut used by the CERES experiment for $\mathrm{S}-\mathrm{Au}$ collisions.

It is not unreasonable [31 to contemplate the possibility of a strong additional source of charm in nucleus-nucleus collisions. However, an enhancement by a factor of 150 would be needed to describe the present CERES 


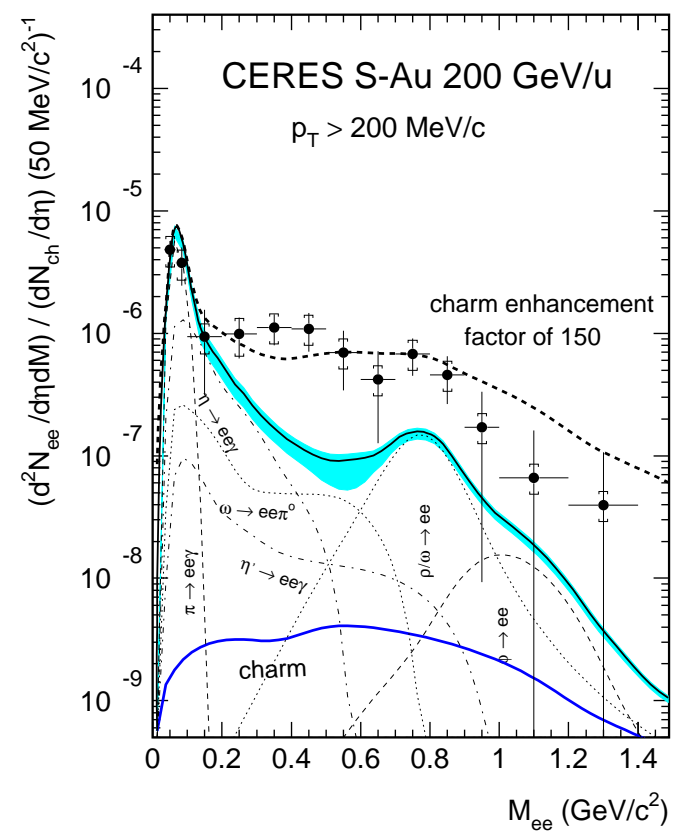

Figure 11: Invariant mass spectrum of $e^{+} e^{-}$pairs measured by CERES in $200 \mathrm{GeV} /$ nucleon $\mathrm{S}-\mathrm{Au}$ collisions. The solid line gives the contribution expected from charm production in the standard scenario. The dashed line results from adding to the hadron decay background a charm contribution enhanced by a factor of 150 .

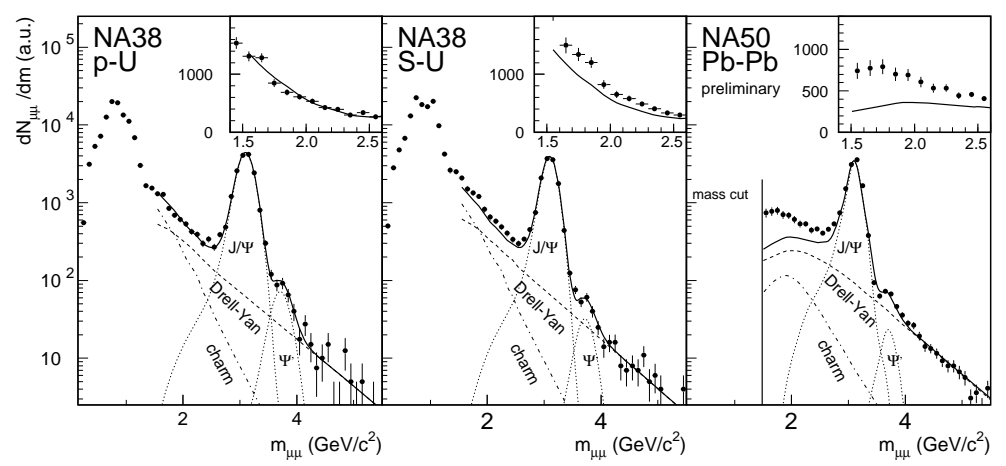

Figure 12: Dimuon spectra from $\mathrm{p}-\mathrm{U}, \mathrm{S}-\mathrm{U}$, and $\mathrm{Pb}-\mathrm{Pb}$ collisions (NA38/NA50 Collaboration). The S-U spectrum excludes charm enhancement by more than a factor of 3 . The figure was taken from 32 and is based on results from [33]. 
data. The result of such an enhancement is shown as the dashed line in Fig. 11. Though it roughly explains the overall dilepton yield, it predicts a flatter mass distribution than is observed resulting in an overestimate of the yield at high masses. The results for dimuon production obtained by the NA38/NA50 Collaboration are shown in Fig. 12 where also the predictions for the charm and Drell-Yan continuum are indicated. Based on these results it is clear that charm enhancement factors larger than 3 would lead to inconsistencies with the S-U dimuon data.

\section{Conclusions}

In summary, the level of charm production in p-p and p-A collisions in the energy range $\sqrt{s} \sim 20-40 \mathrm{GeV}$ is now reasonably well measured. The trends in the data are well reproduced by leading order perturbative QCD calculations performed using the code PYTHIA with $\left\langle k_{\mathrm{T}}^{2}\right\rangle=1 \mathrm{GeV}^{2} / c^{2}$. The calculations were extrapolated to nucleus-nucleus collisions by scaling with the geometrically allowed number of nucleon-nucleon collisions. We have used these calculations to determine the dilepton pair contribution expected from associated $c \bar{c}$ production. In the mass region below the vector mesons $\rho, \omega$ and $\phi$ this contribution is negligible compared to the yield arising from decays of light mesons for all collision systems investigated in this paper. For S-Au collisions the prediction is two orders of magnitude below the experimentally observed $e^{+} e^{-}$continuum. Since any possible enhancement of charm production in nuclear collisions is limited by $\mu^{+} \mu^{-}$data in the mass region above the $\phi$ to less than a factor of 3, any explanation of the observed anomalous low mass pair production based on charm production can be ruled out.

We thank George Ginther, Karl Harrison and Thomas Ullrich for useful discussions while collecting the charm cross section measurements from different experiments. We are grateful to H. G. Fischer for helpful and provocative remarks about charm production and to W. Geist, M. Mangano and A. Morsch for helpful discussions.

\section{References}

[1] C. Lourenço, QM96, Nucl. Phys. A610 (1996) 552c.

[2] T. Sjöstrand, Comp. Phys. Comm. 82 (1994) 74.

[3] C. Lourenço et al. (NA38 Coll.), QM93, Nucl. Phys. A566 (1994) 77c. 
[4] M.A. Mazzoni et al. (HELIOS-3 Coll.), QM93, Nucl. Phys. A566 (1994) $95 \mathrm{c}$.

[5] A. Shor, Proceedings of the International Workshop on Quark Gluon Plasma Signatures, Strasbourg, 1990.

[6] G. Agakichiev et al. (CERES Coll.), Phys. Rev. Lett. 75 (1995) 1272.

[7] S. Frixione et al., CERN-TH/97-16 (hep-ph/9702287) (to be published in Heavy Flavours II, ed. by A.J. Buras and M. Lindner, World Scientific).

[8] R.M. Barnett et al. (PDG), Phys. Rev. D54 (1996) 1.

[9] M. Aguilar-Benitez et al. (NA16 Coll.), Phys. Lett. B135 (1984) 237.

[10] M. Aguilar-Benitez et al. (NA27 Coll.), Z. Phys. C31 (1986) 491;

Z. Phys. C40 (1988) 321.

[11] R. Ammar et al. (E743 Coll.), Phys. Rev. Lett. 61 (1988) 2185.

[12] K. Kodama et al. (E653 Coll.), Phys. Lett. B263 (1991) 573;

Phys. Lett. B284 (1992) 461.

[13] M.J. Leitch et al. (E789 Coll.), Phys. Rev. Lett. 72 (1994) 2542.

[14] G.A. Alves et al. (E769 Coll.), Phys. Rev. Lett. 77 (1996) 2388.

[15] S. Barlag et al. (NA32 Coll.), Z. Phys. C39 (1988) 451;

Z. Phys. C49 (1991) 555.

[16] M. Adamovich et al. (WA92 Coll.), CERN-PPE/96-180.

[17] L. Apanasevich et al. (E706 Coll.), Fermilab-Pub-97/030-E.

[18] G.A. Alves et al. (E769 Coll.), Phys. Rev. Lett. 70 (1993) 722.

[19] H. Plothow-Besch, Comp. Phys. Comm. 75 (1993) 396;

Int. J. Mod. Phys. A10 (1995) 2901.

[20] G.A. Alves et al. (E769 Coll.), Phys. Rev. Lett. 77 (1996) 2392.

[21] W. Bacino et al., Phys. Rev. Lett. 43 (1979) 1073.

[22] H.G. Fischer and W.M. Geist, Z. Phys. C19 (1983) 159.

[23] B.L. Combridge, Nucl. Phys. B151 (1979) 429. 
[24] A. Chilingarov et al., Nucl. Phys. B151 (1979) 29.

[25] T. Åkesson et al. (HELIOS-1 Coll.), Z. Phys. C68 (1995) 47.

[26] M. Masera et al. (HELIOS-3 Coll.), Nucl. Phys. A590 (1995) 93c;

I. Kralik, Slovak Academy of Sience, Kosice, Ph.D. Thesis (1995).

[27] K.J. Eskola et al. Nucl. Phys. B323 (1989) 37.

[28] M.A. Preston and R.K. Bhaduri, "Structure of the Nucleus", AddisonWesley 1975, Chapter 4.

[29] E.M. Aitala et al. (E791 Coll.) Phys. Lett. B371 (1996) 157.

[30] D. Antreasyan et al., Phys. Rev. D19 (1979) 764.

[31] C.Y. Wong and Z.Q. Wang, Phys. Lett. B367 (1996) 50.

[32] A. Drees, QM96, Nucl. Phys. A610 (1996) 536c.

[33] E. Scomparin et al. (NA50 Coll.), QM96, Nucl. Phys. A610 (1996) 331c. 